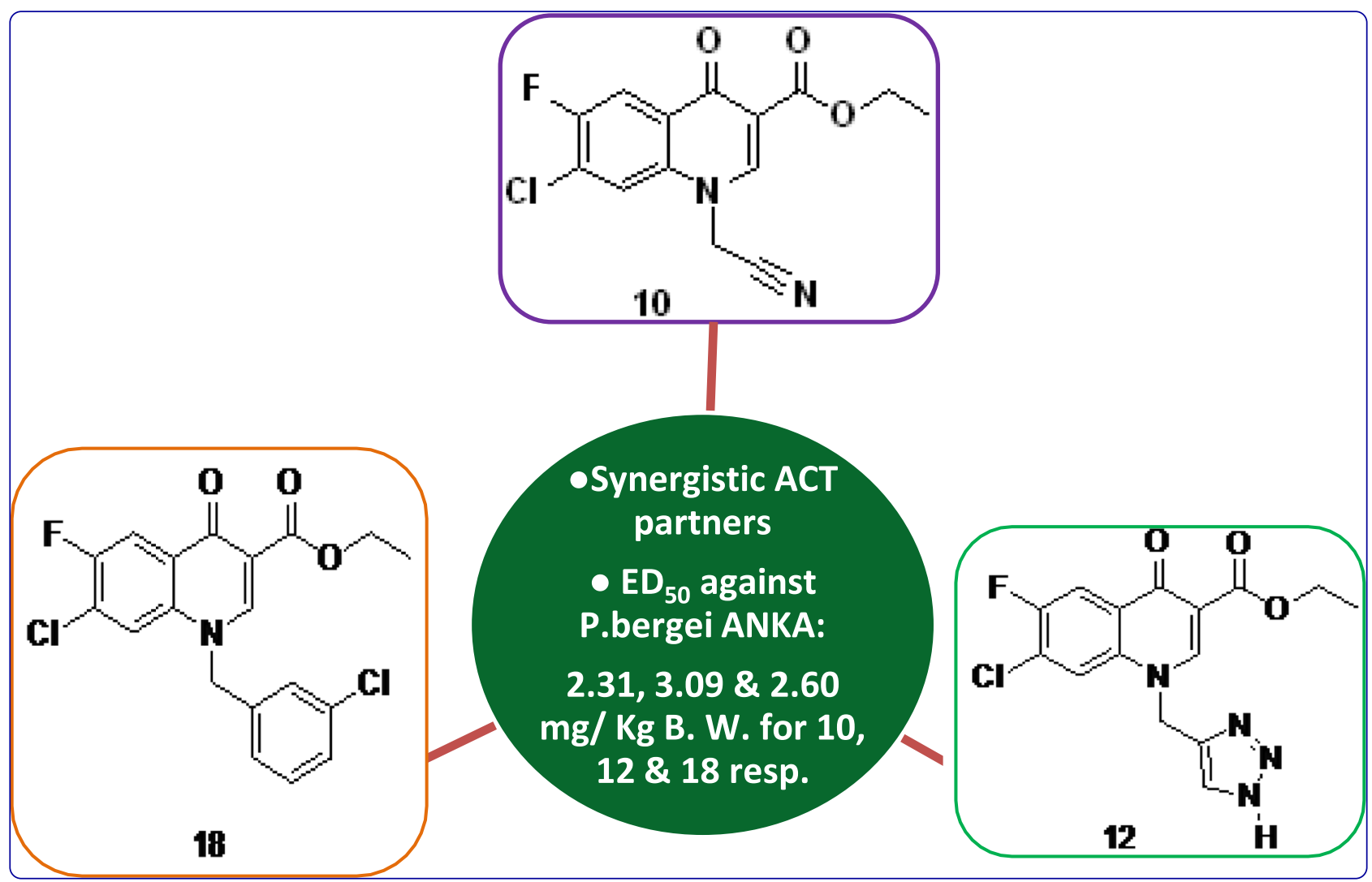

In vitro synergistic effect of fluoroquinolone analogues in combination with artemisinin against Plasmodium falciparum; their antiplasmodial action in rodent malaria model

Agarwal et al. 


\title{
In vitro synergistic effect of fluoroquinolone analogues in combination with artemisinin against Plasmodium falciparum; their antiplasmodial action in rodent malaria model
}

\author{
Drishti Agarwal ${ }^{1,3}$, Manish Sharma², Sandeep K Dixit ${ }^{1}$, Roshan K Dutta ${ }^{3}$, Ashok K Singh ${ }^{2}$, Rinkoo D Gupta ${ }^{3}$ \\ and Satish K Awasthi ${ }^{*}$
}

\begin{abstract}
Background: Emergence of drug-resistant parasite strains has surfaced as a major obstacle in attempts to ameliorate malaria. Current treatment regimen of malaria relies on the concept of artemisinin-based combination therapy (ACT).

Methods: Fluoroquinolone analogues, compounds 10,12 and 18 were investigated for their anti-malarial interaction in combination with artemisinin in vitro, against Plasmodium falciparum 3D7 strain, employing fixed-ratio combination isobologram method. In addition, the efficacy of these compounds was evaluated intraperitoneally in BALB/c mice infected with chloroquine-resistant Plasmodium berghei ANKA strain in the Peters' four-day suppressive test.
\end{abstract}

Results: Promising results were obtained in the form of synergistic or additive interactions. Compounds 10 and 12 were found to have highly synergistic interactions with artemisinin. Antiplasmodial effect was further verified by the convincing $\mathrm{ED}_{50}$ values of these compounds, which ranged between 2.31 and 3.09 ( $\mathrm{mg} / \mathrm{kg} \mathrm{BW}$ ).

Conclusions: In vivo studies substantiated the potential of the fluoroquinolone derivatives to be developed as synergistic partners for anti-malarial drug combinations.

Keywords: Fluoroquinolone derivatives, artemisinin, isobologram, antiplasmodial activity

\section{Background}

Drug-resistant malaria has emerged as the most undefiable obstacle in the battle against this deadly disease $[1,2]$. Artemisinin and its analogues, once regarded as the most powerful drugs that cure chloroquine-resistant Plasmodium falciparum infections, have also fallen to resistance [3-6]. Therefore, the need of the hour is to ward off the deployment of artemisinin and its analogues as monotherapy, to support WHO's resolution of advocating artemisinin-based combination therapy (ACT), and ensure their methodical and practicable implementation in all afflicted areas. As the available ACT is only a handful, there is tremendous possibility of the selection of Plasmodium strains with acquired resistance

\footnotetext{
* Correspondence: satishpna@gmail.com

${ }^{1}$ Chemical Biology Laboratory, Department of Chemistry, University of Delhi, Delhi 110007, India

Full list of author information is available at the end of the article
}

towards them. Therefore, the current focus should be directed towards devising alternative ACT. The underlying mechanism behind the therapeutic effect of artemisininbased combinations is that the artemisinin component rapidly and effectively wipes out most of the parasites, while those that remain are successively annihilated by a high concentration of the partner drug [7]. The efficacy and short half life (< one hour) of the artemisinin component confers protection against development of drug resistance. The long half life companion drug is required to ensure no parasite is left unperturbed. In this manner, the probability that mutant parasites survive and emerge after co-administration of these two drugs is very low.

In spite of the availability of several potent drugs as partners in ACT, quinolones are one of the cardinal classes as they can target both the blood and liver parasite stages [8]. The current status of quinolones as anti-malarials can be traced back to 1962 when Lesher et al. [9] discovered 
nalidixic acid as a by-product of the synthesis of the antimalarial drug, chloroquine. This discovery paved the way for further development of a vast array of quinolone compounds, along with those in clinical use [10,11]. Presently, primaquine and atovaquone are the only anti-malarials available commercially that target liver stage parasites as well $[12,13]$. The first quinolone identified to possess activity against multiple parasite forms was endochin, 4 (1H)-quinolone compound, in avian malaria models [14]. A long time after its discovery back in the 1940s, other fluoroquinolones, such as norfloxacin, ciprofloxacin, pefloxacin, grepafloxacin, trovafloxacin, enoxacin, and clinofloxacin were evaluated against the malaria parasite in vitro $[9,15,16]$ and in vivo [17-19]. Although these common antibiotics were found efficacious against both chloroquine-sensitive and -resistant parasites, highly effective concentrations and prolonged treatment regimen (14 days) have restricted their use as sole therapy. These findings support further screening of newer fluoroquinolone compounds as partner drugs.

The synthesis of two series of fluoroquinolone analogues has been reported previously, amongst which several compounds exhibited significant anti-malarial activity, with very low to negligible toxicity [20]. These are substituted fluoroquinolones with normal and branched chain alkyl groups as well as some polar groups such as $-\mathrm{OH},-\mathrm{CN}$ and $-\mathrm{C} \equiv \mathrm{CH}$ etc. In the present study, three most active compounds (Figure 1) from the aforementioned series were selected, which yielded least inhibitory concentrations, i.e., inhibited the parasite multiplication rate to $50 \%$ $\left(\mathrm{IC}_{50}\right)$ at concentrations of $<3 \mu \mathrm{g} / \mathrm{ml}(2.56 \pm 0.30,1.33 \pm$ 0.67 and $2.73 \pm 0.23 \mu \mathrm{g} / \mathrm{ml} \pm \mathrm{SE}$ (equivalent to $8.69,3.79$ and $6.93 \mu \mathrm{M})$, and exhibited in vitro host cell cytotoxicity $\mathrm{IC}_{50}$ values of $142.81,171.37$ and $129.24 \mu \mathrm{M}$ for compounds 10, 12 and 18, respectively. These values are strikingly lower than that of the fluoroquinolones in clinical use. While the reported $\mathrm{IC}_{50}$ values $(\mu \mathrm{M})$ of ciprofloxacin, clinafloxacin, and norfloxacin against 3D7 strain of $P$. falciparum in vitro are 27.77, 37.45 and 53.86, respectively, those of enoxacin and ofloxacin rise up to 121.13 and 152.10. Therefore, it was considered interesting to investigate the fixed-ratio combinatorial interactions of each of these three novel fluoroquinolone derivatives with artemisinin, for treating the erythrocytic stages of $P$. falciparum strain 3D7. A modified isobologram method [21] was followed to assess the synergistic, antagonistic or additive interactions of the combinations. Additionally, on account of their convincing antiplasmodial activity under in vitro conditions, it was imperative to assess their efficacy in vivo, employing a rodent malaria model.

\section{Methods}

\section{Antiplasmodial interaction assay of artemisinin and fluoroquinolone analogue combinations \\ Parasite culture}

Stock culture of malaria parasite P. falciparum 3D7 strain (chloroquine sensitive) was continuously maintained in vitro using a $\mathrm{CO}_{2}$ incubator under low-oxygen concentration (3\%) and high carbon dioxide atmosphere (4\%) along with nitrogen (93\%), incubated at a temperature of $37^{\circ} \mathrm{C}$. The parasites were maintained on $\mathrm{O}^{+}$human red blood cells suspended in a complete culture medium. Each litre of RPMI-1640 aqueous culture medium was prepared with 10.4 g of powdered RPMI-1640 (with glutamine but without sodium bicarbonate), 5.94 $\mathrm{g}$ of HEPES buffer, $1 \mathrm{~g}$ of dextrose and $40 \mathrm{mg}$ of gentamicin. Complete medium was constituted just before use by adding sterile $5 \%$ sodium bicarbonate at the rate of $4 \mathrm{ml}$ per $96 \mathrm{ml}$, and supplemented with $10 \%(\mathrm{v} / \mathrm{v})$ pooled $\mathrm{O}^{+}$human serum. Infected erythrocytes were suspended in this culture media initiated at a haematocrit value of $5 \%$ and parasitemia was kept between 2 and $4 \%$ with sub-culturing done beyond 5\%. Medium was changed once a day and percentage parasitemia was monitored using Giemsa stained slides.

\section{Stock solution of compounds}

Artemisinin (Sigma Aldrich, USA) was prepared in DMSO to get the stock solution of $1 \mathrm{mg} / \mathrm{ml}$ strength. Compounds 10, 12 and 18 were synthesized according to the procedure described by Dixit et al. [20] and each compound was made to strength of $1 \mathrm{mg} / \mathrm{ml}$ stock solution in DMSO. The stock solutions were diluted on the day of experiment to get the desired concentrations for each compound. The highest amount of DMSO in diluted concentrations was $0.125 \%$, and had no effect on parasite growth.

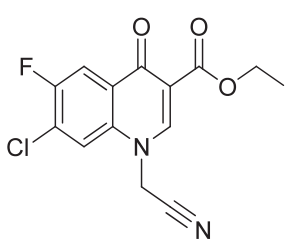

10

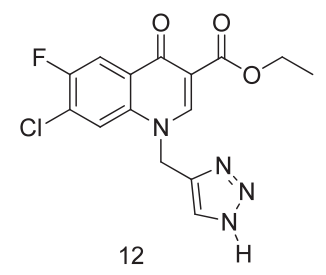

12

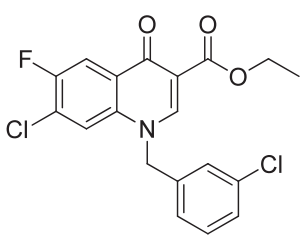

18

Figure 1 Chemical structures of compounds 10, 12 and 18. 


\section{Preparation of fixed-ratio combinations}

In each combination assay, two compounds (Compound $\mathrm{A}$, artemisinin and Compound $\mathrm{B}$, a fluoroquinolone derivative or norfloxacin) were combined in four fixed ratios (4:1, 3:2, 2:3, and 1:4). Approximately eight-fold $\mathrm{IC}_{50} \mathrm{com}$ pound concentration of the respective compound $\mathrm{A}$ or $\mathrm{B}$ was taken as $100 \%$ (calculated to be $32 \mathrm{nM}, 69.50 \mu \mathrm{M}$, $30.34 \mu \mathrm{M}, 55.40 \mu \mathrm{M}$, and $430.88 \mu \mathrm{M}$ for artemisinin, compound 10, compound 12, compound 18 and norfloxacin, respectively), so that $\mathrm{IC}_{50}$ of the individual compound falls in between third and fourth two-fold serial dilution.

\section{Plate preparation for antiplasmodial interaction assay}

Compound dilutions of each combination solution were made in sterile, flat-bottomed, 96-well tissue culture plates as described by Fivelman et al. [21]. Six times two-fold serial dilution was done for each combination in triplicate. Each well contained a total volume of $200 \mu \mathrm{l}$ of complete culture medium with or without compound and presynchronized infected red blood cells (1\% parasitemia at $2.5 \%$ haematocrit). Control cultures (without compound) were maintained on the same plate in triplicate. Two 96well plates (for six combinations) were used for each combination experiment. The plates were stacked in a $\mathrm{CO}_{2}$ incubator and incubated at $37^{\circ} \mathrm{C}$ for 48 hours.

\section{Slide preparation, staining and assessment}

After 48-hour incubation, thin blood smear slides were prepared, air dried, methanol fixed, and stained in Giemsa solution for $40 \mathrm{~min}$. After staining, slides were removed from coupling jar, washed in running tap water and air dried. The Giemsa-stained slides were examined for counting the number of parasites in random adjacent microscopic fields, equivalent to about 4,000 erythrocytes at 1,000 $\times$ magnification. Per cent parasitemia was calculated. Reproducibility of counts was checked by two other readers to maintain the quality control.

\section{Isobologram preparation and data analysis}

For each combination assay, $\mathrm{IC}_{50}$ was calculated from two sets of concentration response graphs, each containing compound alone curve and four combination curves.

The sum FIC of each combination ratio of two combined compounds shows that the drug-drug schizontocidal interaction between them [22] was determined by the following equation:

$$
\text { Sum FIC }\left(\sum \text { FIC }\right)=\frac{\text { IC50 of A in mixture }}{\text { IC50 of A alone }}+\frac{\text { IC50 of B in mixture }}{\text { IC50 of B alone }}
$$

$\Sigma$ FIC $<1$ represents synergism, $\Sigma$ FIC $>=1$ and $<2$ represents additive interaction, $\Sigma F I C>=2$ and $<4$ represents slight antagonism while $\Sigma$ FIC $>=4$ represents marked antagonism [23-25]. Mean FICs of the combinations were compared by frequency distribution using GraphPad
Prism 5, to define if a compound was superior to the other one, when combined with artemisinin.

\section{In vivo efficacy of fluoroquinolone analogues using rodent malaria model}

Evaluation of the curative potential of the fluoroquinolone derivatives was done using the method described by Ryley and Peters, 1970 (rodent malaria four-day suppressive test; Peters' four-day suppressive test) [26,27]. Rodent malaria parasite Plasmodium berghei ANKA was used.

\section{Experimental animals}

Immuno-compromised BALB/c inbred albino mice (25$30 \mathrm{~g}$ ) of the male sex were obtained from the Animal Facility Centre of the Department of Zoology, University of Delhi. The animals were fed ad libitum with standard feed and had free access to water. They were maintained under standard conditions of humidity, temperature $\left(25^{\circ} \mathrm{C}\right)$ and 12 hours light/darkness cycles. The animals were acclimatized for two weeks before the commencement of the study and were ensured to exclude all zoonotic agents.

\section{Test procedure}

Day 0: Heparinized blood was withdrawn from an infected donor mouse with approximately $25-30 \%$ parasitemia, and diluted in $1 \mathrm{x}$ PBS to $10^{8}$ parasitized erythrocytes per mL. An aliquot of $0.2 \mathrm{~mL}\left(=2 \times 10^{7}\right.$ parasitized erythrocytes $)$ of this suspension was injected intraperitoneally (ip) into experimental groups of five mice each. One to three hours post-infection, the experimental groups were treated with varying doses of each of the test compounds $(0.5,1,10$, $25 \mathrm{mg} / \mathrm{kg} \mathrm{BW}$ ) by the ip route. Each compound was made to strength of $5 \mathrm{mg} / \mathrm{ml}$ stock solution in 10\% DMSO and administered according to desired concentration and individual body weight. Artemisinin was given to the standard drug group and $0.2 \mathrm{~mL}$ of normal saline to the negative control group.

Day 1, 2 and 3: 24 hours, 48 hours and 72 hours postinfection, the experimental groups of mice were treated again with the same dose and by the same route as on day 0 .

Day 4: 24 hours after the last treatment (i.e., 96 hours post-infection), blood was drawn from the tail region of mice and smears were prepared. These were stained with Giemsa for microscopic analysis by counting four fields of approximately 500 erythrocytes per slide, for five replicates of each sample, to determine the parasitemia percentage and hence assess the anti-malarial efficacy of the test compounds. Differences in parasitemia percentage between treated groups and untreated animals were analysed by a one-way ANOVA test using IBM SPSS Statistics 16.0 and differences considered significant if $\mathrm{P}<0.05$. Furthermore, the difference between the mean value of the negative control group (taken as 100\%) and those of the 
experimental groups was calculated and expressed as per cent inhibition (= activity) using the equation below and hence $\mathrm{ED}_{50}$ value was calculated graphically.

Per cent inhibition $($ activity $)=100-($ Mean parasitemia/control $) \times 100$

Untreated control mice typically died approximately one week after infection. Treated mice were observed for a period of 30 days, and the survival time (in days) was recorded. The mean survival time was calculated in comparison to untreated (Normal saline) and standard drug (artemisinin) treated groups. Differences in survival time between treated groups and untreated animals were analysed by Log-rank (Mantel-Cox) test using GraphPad Prism 5 and differences considered significant if $\mathrm{P}<0.005$. Observations concerning adverse effects due to the compounds were recorded.

\section{$\mathrm{LD}_{50}$ Test}

$\mathrm{LD}_{50}$ test was carried out on BALB/c mice using different dosages of various compounds: 50, 100, 200, 500, 600,800 and $1000 \mathrm{mg} / \mathrm{kg} \mathrm{BW}$ ip and the animals were observed for 7 days. Therapeutic Index (TI) values were determined by the formula:

$$
\text { Therapeutic Index }(\mathrm{TI})=\frac{\text { Median lethal dose }\left(\mathrm{LD}_{50}\right)}{\text { Median effective dose }\left(\mathrm{ED}_{50}\right)}
$$

\section{Results}

Antiplasmodial interactions between artemisinin and fluoroquinolone analogues

Various substituted fluoroquinolones were synthesized by the earlier reported procedure [20]. The pharmacophore of fluoroquinolones shows striking similarity with chloroquine, which has been the forerunner for malaria

Table 1 Interaction between artemisinin and various fluoroquinolones analogs (compounds 10, 12 and 18) against Plasmodium falciparum (3D7 strain) at six different preparations

\begin{tabular}{|c|c|c|c|}
\hline Combination solution (Ratio A:B) & Drug A (Artemisinin) Mean $\mathrm{FIC}_{50} \pm \mathrm{SE}^{\mathrm{a}}$ & Drug B (Compound-10) Mean FIC $\mathrm{F}_{50} \pm \mathrm{SE}^{\mathrm{a}}$ & $\Sigma$ FICs, interaction ${ }^{\mathbf{b}}$ \\
\hline $1(5: 0)$ & $1.02 \pm 0.04$ & 0 & \\
\hline $2(4: 1)$ & $0.61 \pm 0.01$ & $0.15 \pm 0.02$ & 0.76 SYN \\
\hline $3(3: 2)$ & $0.58 \pm 0.03$ & $0.22 \pm 0.02$ & 0.80 SYN \\
\hline $4(2: 3)$ & $0.48 \pm 0.02$ & $0.53 \pm 0.03$ & $1.01 \mathrm{ADD}$ \\
\hline $5(1: 4)$ & $0.17 \pm 0.03$ & $0.41 \pm 0.03$ & 0.58 SYN \\
\hline $6(0: 5)$ & 0 & $0.93 \pm 0.04$ & \\
\hline Combination solution (Ratio A:B) & Drug A (Artemisinin) Mean $\mathrm{FIC}_{50} \pm \mathrm{SE}^{\mathrm{a}}$ & Drug B (Compound-12) Mean $\mathrm{FIC}_{50} \pm \mathrm{SE}^{\mathrm{a}}$ & $\Sigma$ FICs, interaction ${ }^{b}$ \\
\hline $1(5: 0)$ & $1.0 \pm 0.02$ & 0 & \\
\hline $2(4: 1)$ & $0.63 \pm 0.03$ & $0.15 \pm 0.003$ & 0.78 SYN \\
\hline $3(3: 2)$ & $0.60 \pm 0.03$ & $0.23 \pm 0.02$ & 0.83 SYN \\
\hline $4(2: 3)$ & $0.50 \pm 0.02$ & $0.77 \pm 0.03$ & 1.27 ADD \\
\hline $5(1: 4)$ & $0.25 \pm 0.004$ & $0.32 \pm 0.01$ & 0.56 SYN \\
\hline $6(0: 5)$ & 0 & $1.11 \pm 0.08$ & \\
\hline Combination solution (Ratio $\mathrm{A}: \mathrm{B}$ ) & Drug A (Artemisinin) Mean $\mathrm{FIC}_{50} \pm \mathrm{SE}^{\mathrm{a}}$ & Drug B (Compound 18) Mean $\mathrm{FIC}_{50} \pm \mathrm{SE}^{\mathrm{a}}$ & $\Sigma$ FICs, interaction ${ }^{b}$ \\
\hline $1(5: 0)$ & $0.97 \pm 0.07$ & 0 & \\
\hline $2(4: 1)$ & $0.58 \pm 0.02$ & $0.16 \pm 0.01$ & 0.73 SYN \\
\hline $3(3: 2)$ & $0.55 \pm 0.03$ & $0.47 \pm 0.03$ & $1.02 \mathrm{ADD}$ \\
\hline $4(2: 3)$ & $0.49 \pm 0.04$ & $0.66 \pm 0.03$ & $1.15 \mathrm{ADD}$ \\
\hline $5(1: 4)$ & $0.22 \pm 0.01$ & $0.33 \pm 0.02$ & 0.55 SYN \\
\hline $6(0: 5)$ & 0 & $0.97 \pm 0.08$ & \\
\hline Combination solution (Ratio $\mathrm{A}: \mathrm{B}$ ) & Drug A (Artemisinin) Mean $\mathrm{FIC}_{50} \pm \mathrm{SE}^{\mathrm{a}}$ & Drug B (Norfloxacin) Mean $\mathrm{FIC}_{50} \pm \mathrm{SE}^{\mathrm{a}}$ & $\Sigma$ FICs, interaction ${ }^{\mathbf{b}}$ \\
\hline $1(5: 0)$ & $1.13 \pm 0.06$ & 0 & \\
\hline $2(4: 1)$ & $0.91 \pm 0.03$ & $0.45 \pm 0.02$ & 1.36 ADD \\
\hline $3(3: 2)$ & $0.29 \pm 0.02$ & $0.52 \pm 0.04$ & 0.81 SYN \\
\hline $4(2: 3)$ & $0.66 \pm 0.02$ & $0.87 \pm 0.03$ & $1.53 \mathrm{ADD}$ \\
\hline $5(1: 4)$ & $0.14 \pm 0.01$ & $0.60 \pm 0.02$ & 0.74 SYN \\
\hline $6(0: 5)$ & 0 & $0.98 \pm 0.04$ & \\
\hline
\end{tabular}

${ }^{\mathrm{a} S t a n d a r d}$ error $(\mathrm{n}=3){ }^{\mathrm{b}} \mathrm{ADD}$, additive; SYN, synergistic. 

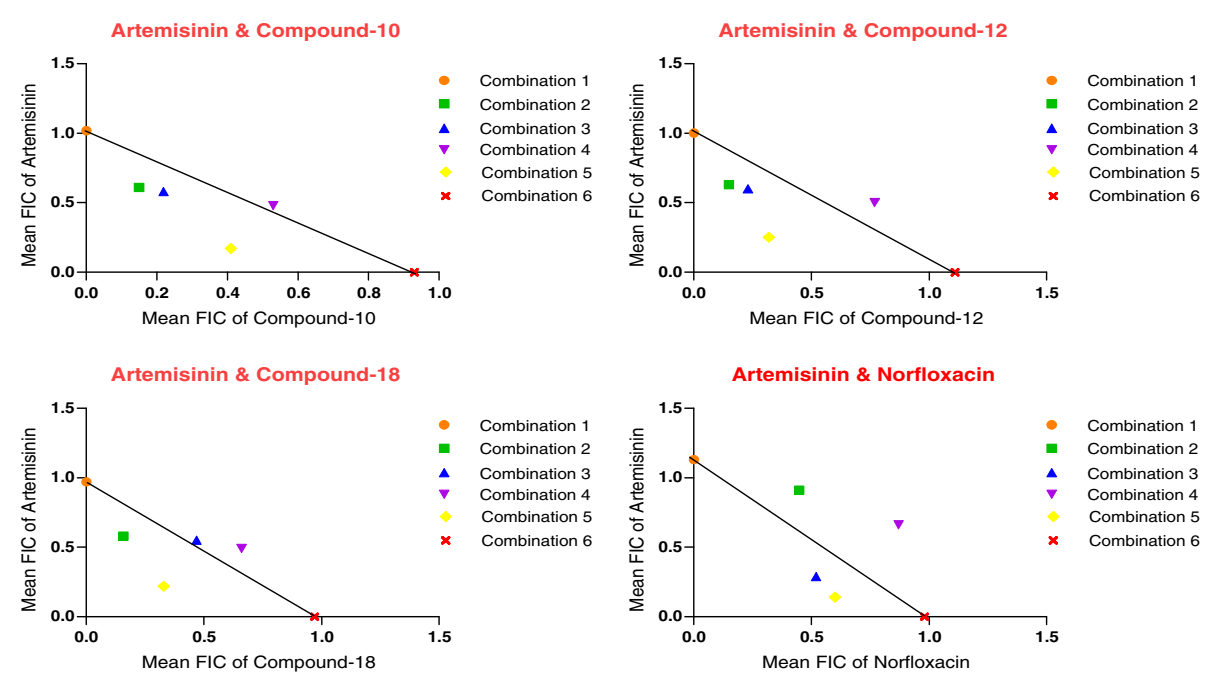

Figure 2 Isobolograms showing interaction between artemisinin and fluoroquinolone derivatives; artemisinin and norfloxacin against Plasmodium falciparum 3D7 strain.

treatment for the past 50 years [28]. Both fluoroquinolones and choloroquine contain chlorine at position 7. A vast array of fluoroquinolones have been investigated and henceforth proven effective against P. falciparum. Here, three substituted fluoroquinolone compounds, 10, 12 and 18 were chosen from previous in vitro study [20], and were tested in combination with artemisinin in vitro against $P$. falciparum chloroquine-sensitive 3D7 strain, using norfloxacin as the standard drug. These compounds were found to have synergistic and additive drug-drug interactions. In every combination assay $\mathrm{IC}_{50}$ was determined from two sets of drug response curves obtained from each replicate, each set representing four combination curves and a curve of drug/compound alone. Mean $\mathrm{FIC}_{50}$ values derived from these curves are tabulated in Table 1, for each fluoroquinolone derivative combination with artemisinin, and combination of norfloxacin with artemisinin. Sum of FICs are presented in isobolograms (Figure 2). The isobolograms show that anti-malarial interaction of the fluoroquinolone derivatives in vitro with artemisinin is not antagonistic. Compound $\mathbf{1 0}$ in combination with artemisinin shows synergistic antiplasmodial interaction in three of the four fixed-ratio combinations evaluated and additive in the remaining one. Similarly the combination of compound 12 and artemisinin displays synergistic interaction in

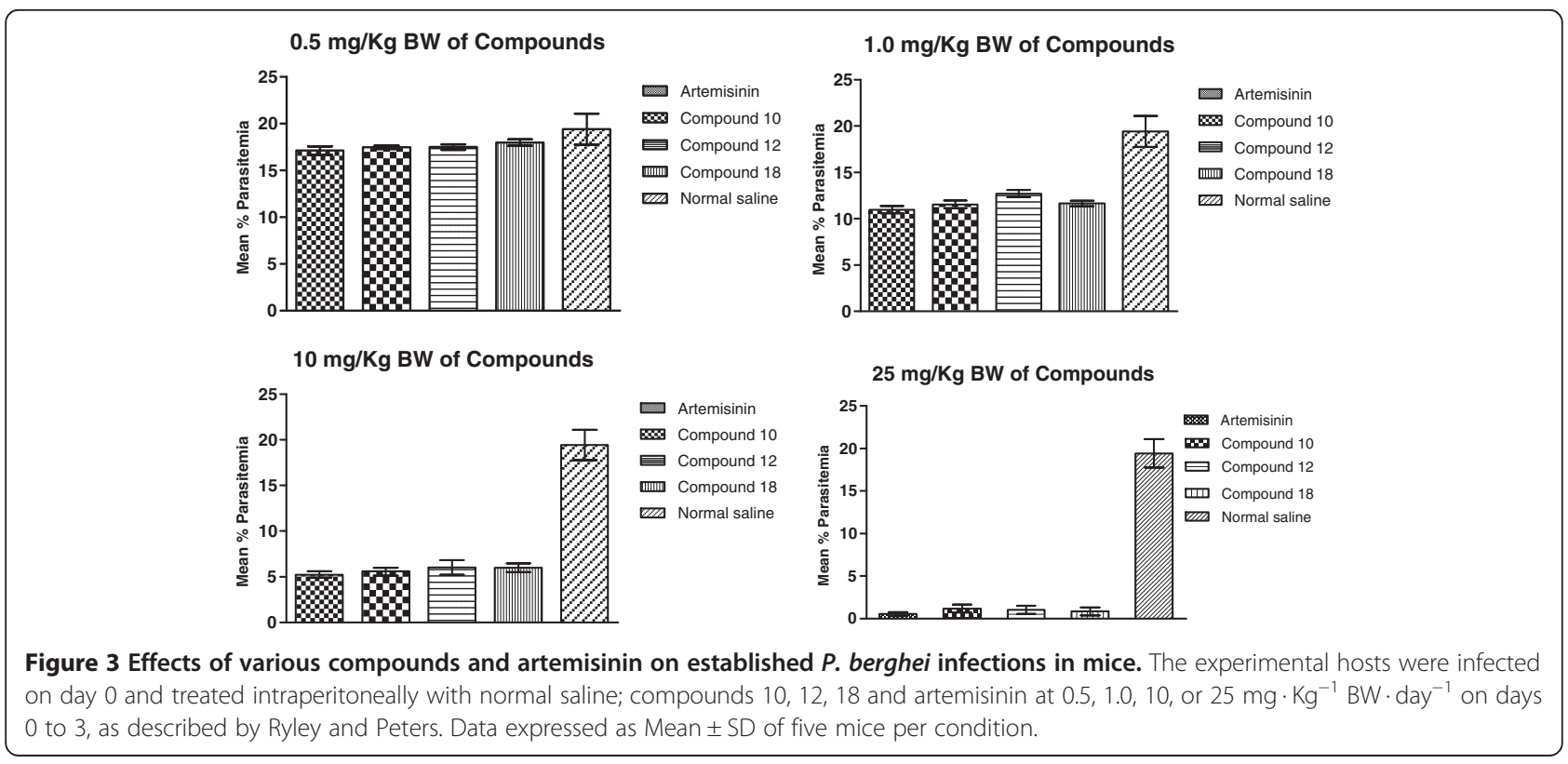


Table 2 Antiplasmodial activity of fluoroquinolone derivatives against Plasmodium berghei strain ANKA

\begin{tabular}{ll}
\hline Compound & Mean $\mathrm{ED}_{\mathbf{5 0}}(\mathbf{m g} / \mathbf{k g ~ B W}) \pm \mathbf{S E}^{\mathbf{a}}$ \\
\hline 10 & $2.31 \pm 0.19$ \\
12 & $3.09 \pm 0.22$ \\
18 & $2.60 \pm 0.18$ \\
Artemisinin & $1.72 \pm 0.15$ \\
\hline
\end{tabular}

${ }^{\mathrm{a} S t a n d a r d}$ error $(n=5)$.

three combinations and additive in one. Interaction of artemisinin with compound $\mathbf{1 8}$ was found to be synergistic in two combinations, while additive in the other two. The standard drug used in the study, norfloxacin, when combined with artemisinin shows synergistic interaction in two combinations, while additive in the remaining two. Combination of compound 10 with artemisinin tended most towards synergism, with respect to all the other compounds (Mean $\Sigma F I C \pm S D=0.788 \pm 0.177$ ), as observed by frequency distribution. Combinations of all fluoroquinolone analogues were superior to that of norfloxacin (Mean $\Sigma \mathrm{FIC} \pm \mathrm{SD}=1.11 \pm 0.394)$, which tended slightly towards antagonism.

In principle, components of anti-malarial combinations should target different metabolic pathways. This condition is being theoretically met in the combinations being evaluated. The hypothesized mechanisms of action of artemisinin include haem alkylation, inhibition of PfATP6 (SERCA-type enzyme), parasite membrane damage [29-31]. On the contrary, fluoroquinolones are the only class of antimicrobial agents that are direct inhibitors of bacterial DNA synthesis. They inhibit two bacterial enzymes: DNA gyrase, particularly the A subunit, and topoisomerase IV, which have essential roles in DNA replication [32]. Plasmodium falciparum contains a functional apicoplast, an organelle of prokaryotic origin. The 27-35 kb circular genome of apicoplast requires bacterial type DNA gyrase for its duplication [33-35]. This is the most likely explanation for inhibitory activity of fluoroquinolones against the parasite, which is being enhanced on interaction with artemisinin either synergistically or additively, analysed using fixedratio isobolograms. However, the exact mode of action of fluoroquinolones against malaria parasites is still ambiguous.

\section{Antiplasmodial activity of synthetic fluoroquinolones against Plasmodium berghei in vivo}

It was observed that there was a reduction in the levels of parasitemia in all the test groups, as well as that of the standard drug (artemisinin) group. However, the reverse was the case for the negative control group, as there was a marked increase in parasitemia level. The in vivo anti-malarial activity of the various test compounds, after conducting Peters' four-day suppressive test, is presented in Figure 3. Results were significant as analysed by ANOVA $(\mathrm{P}<0.05)$. The $\mathrm{ED}_{50}$ values were calculated to be $2.31,3.09,2.60$, and $1.72 \mathrm{mg} / \mathrm{kg}$ BW for each of the compounds 10, 12, 18, and artemisinin, respectively, as indicated in Table 2, and represented graphically in Figure 4. The mean survival time (MST)

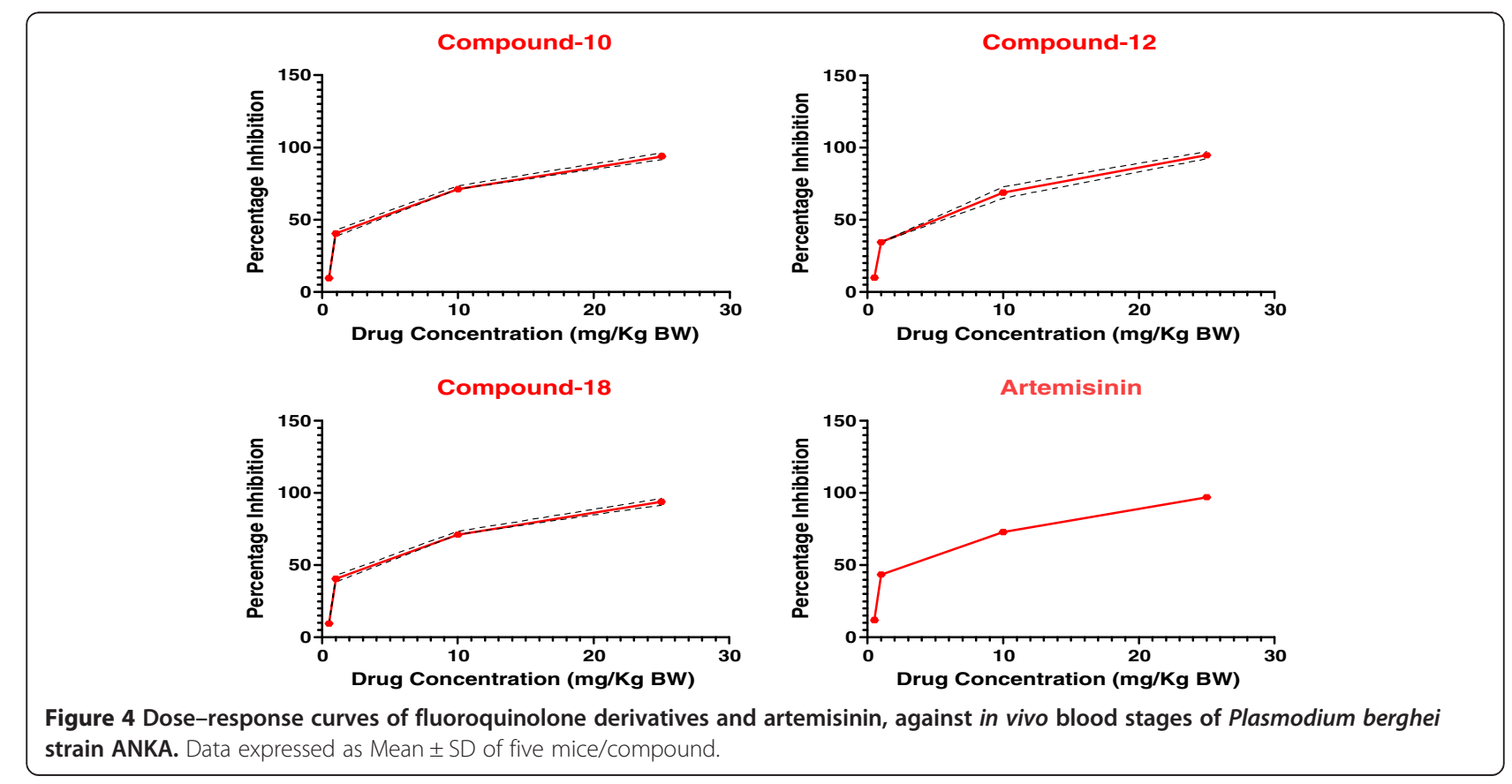



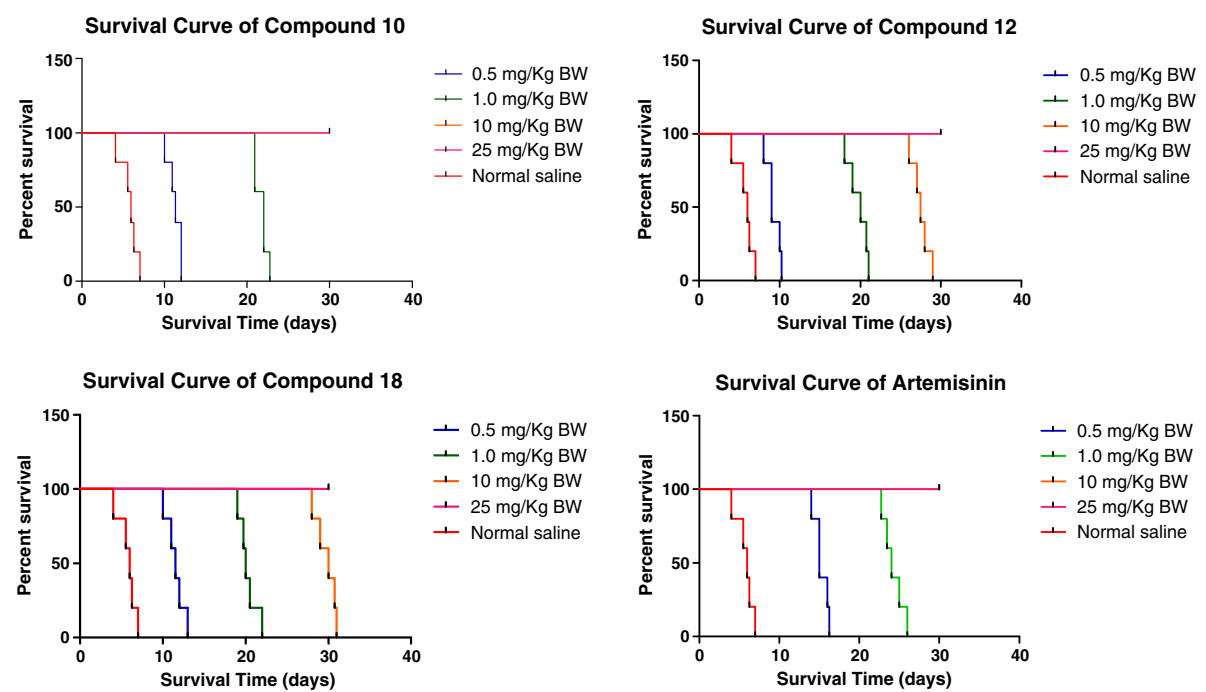

Figure 5 Kaplan Meier survival analysis curves of BALB/c mice, administered drugs once daily ip for four consecutive days $(5$ mice per group). Results between test and control were significant by $P<0.005$ as analysed by Log-rank test.

values of the treated groups were significantly higher than that of control and were comparable to that of the standard drug, artemisinin (Figure 5). The mice treated with varying doses of each of the fluoroquinolone derivatives survived beyond one week, but $0.5 \mathrm{mg} / \mathrm{kg}$ BW treated mice died nine to 12 days post treatment. Artemisinintreated mice on the other hand, survived beyond two weeks in all the groups.

The results show that two out of the three compounds (10 and 18) exhibited appreciable antiplasmodial activity, reflected by their $\mathrm{ED}_{50}$ values, comparable to that of the standard drug, artemisinin. The study on MST demonstrated a dose-dependent increase in the number of days the mice survived in various groups survived postfour-day treatment. MST values for compound $\mathbf{1 0}$ (with lowest $\mathrm{ED}_{50}=2.31 \mathrm{mg} / \mathrm{kg} \mathrm{BW}$ ) were the most proximal to those obtained for artemisinin, while the next to follow was compound $18\left(\mathrm{ED}_{50}=2.60 \mathrm{mg} / \mathrm{kg} \mathrm{BW}\right)$. All the results were significant $(\mathrm{P}<0.005)$ as analysed by Logrank (Mantel-Cox) test. Therefore, they serve as promising candidates for further research. No significant adverse side effects, i.e., physical signs such as gasping for air, loss of appetite, feeling sleepy, or weight loss were observed in compound-treated groups, even at the highest dose administered, indicating that the compounds are well tolerated by the biological system and may be toxic at doses much higher than those required for their therapeutic effects. Thus, compounds $\mathbf{1 0}$ and $\mathbf{1 8}$ could be excellent candidates for combination therapy.

\section{LD50 Test}

$\mathrm{BALB} / \mathrm{c}$ mice died at $1000 \mathrm{mg} / \mathrm{kg} \mathrm{BW}$ of all the compounds and could tolerate $500 \mathrm{mg} / \mathrm{kg} \mathrm{BW}$. However, at
$800 \mathrm{mg} / \mathrm{kg} \mathrm{BW}$, half the population of mice died. Therapeutic indices were determined as 346.32, 258.90, 307.69 for compounds 10, 12 and 18, respectively.

\section{Conclusions}

Substantial evidence has been furnished that the fluoroquinolone analogues under assessment show inhibitory activities against the blood stages of the malaria parasite, with $\mathrm{ED}_{50}$ values in single-digit, micro-molar range. The above analogues have been shown to display a synergistic mode of interaction with artemisinin, in majority of fixed-ratio combinations analysed in vitro. The results of this work further justify the use of novel, synthetic fluoroquinolones in the treatment of malarial infection. It is therefore concluded that compounds 10, 12 and 18 have appreciable anti-malarial activity that can be exploited for the production of modern anti-malarial pharmaceuticals. Drugs that target both the liver and blood stages of malaria are urgently required to reduce the disease's extensive worldwide morbidity and mortality [33]. Hence, it would be highly intriguing to evaluate the possibility of these compounds to target the liver stage parasites and dormant hypnozoites as well, which would strengthen their position as potential, all-purpose anti-malarial drug candidates. To find a lead molecule for drug development, extensive SAR is needed and further investigation in this direction is under progress.

\section{Abbreviations}

ACT: Artemisinin-based combined therapy; RPMI: Roswell Park Memorial Institute (cell culture medium); HEPES: 4-(2-hydroxyethyl)-1-piperazineethanesulfonic acid; $I C_{50}$ : Median inhibition concentration of growth; $\mathrm{ED}_{50}$ : Median effective dose; FIC: Fractional inhibitory concentration; DMSO: Dimethyl sulphoxide, MST, mean survival time; SAR: Structural-activity relationship; BW: Body weight. 


\section{Competing interests}

The authors declare that they have no competing interests.

\section{Authors' contributions}

SKA designed and coordinated the study. SKD performed the synthesis of the evaluated compounds. DA, MS and RKD performed the in vitro antiplasmodial combination assays. DA and MS carried out the in vivo study, arranged the data, conceptualized and prepared the manuscript. AKS and RDG participated in data analysis and presentation. All authors read and approved the final manuscript.

\section{Acknowledgements}

RDG thanks the Department of Science and Technology (SERB/F/1424/2013-14) and South Asian University, New Delhi, India, for financial assistance. SKA is thankful to The University of Delhi, Delhi, India for financial support.

\section{Author details}

${ }^{1}$ Chemical Biology Laboratory, Department of Chemistry, University of Delhi, Delhi 110007, India. ${ }^{2}$ Department of Zoology, University of Delhi, Delhi 110007, India. ${ }^{3}$ Faculty of Life Sciences and Biotechnology, South Asian University, New Delhi 110021, India.

Received: 13 October 2014 Accepted: 14 January 2015

Published online: 05 February 2015

\section{References}

1. Kim Y, Schneider KA. Evolution of drug resistance in malaria parasite populations. Nature Education Knowledge. 2013;4:6.

2. Hastings IM. The origins of antimalarial drug resistance. Trends Parasitol. 2004;20:512-8.

3. WHO. Emergency response to artemisinin resistance in the Greater Mekong subregion: regional framework for action 2013-2015. Geneva: World Health Organization; 2013.

4. Fairhurst RM, Nayyar GM, Breman JG, Hallett R, Vennerstrom JL, Duong S, et al. Artemisinin-resistant malaria: research challenges, opportunities, and public health implications. Am J Trop Med Hyg. 2012;87:231-41.

5. Talisuna AO, Karema C, Ogutu B, Juma E, Logedi J, Nyandigisi A, et al. Mitigating the threat of artemisinin resistance in Africa: improvement of drug-resistance surveillance and response systems. Lancet Infect Dis. 2012;12:888-96.

6. Mita T, Tanabe K. Evolution of Plasmodium falciparum drug resistance: implications for the development and containment of artemisinin resistance. Jpn J Infect Dis. 2012;65:465-75.

7. Peters W. Plasmodium: resistance to antimalarial drugs. Ann Parasitol Hum Comp. 1990;65:103-6.

8. Mahmoudi N, Ciceron L, Franetich JF, Farhati K, Silvie O, Eling W, et al. In vitro activities of 25 quinolones and fluoroquinolones against liver and blood stage Plasmodium spp. Antimicrob Agents Chemother. 2003;47:2636-9.

9. Lesher GY, Froelich EJ, Gruett MD, Bailey JH, Brundage RP. 1, 8-Naphthyridine derivatives: a new class of chemotherapeutic agents. J Med Pharm Chem. 1962;91:1063-5.

10. Andriole VT. The quinolones: past, present, and future. Clin Infect Dis. 2005;41:113-9.

11. Beteck RM, Smit FJ, Haynes RK, N'Da DD. Recent progress in the development of anti-malarial quinolones. Malar J. 2014;13:339.

12. Baird JK, Hoffman SL. Primaquine therapy for malaria. Clin Infect Dis. 2004;39:1336-45

13. Rodrigues T, Prudencio M, Moreira R, Mota MM, Lopes F. Targeting the liver stage of malaria parasites: a yet unmet goal. J Med Chem. 2012;55:995-1012.

14. Salzer W, Andersag THH. A new type of compounds active against avian malaria. Chem Ber. 1948:81:12-9.

15. Krishna S, Davis TME, Chan PCY, Wells RA, Robson KJH. Ciprofloxacin and malaria. Lancet. 1988;1:1231-2.

16. Hamzaha J, Skinner-Adams T, Davis TME. In vitro antimalarial activity of trovafloxacin, a fourth-generation fluoroquinolone. Acta Trop. 2000;74:39-42.

17. Sarma PS. Norfloxacin: a new drug in the treatment of falciparum malaria. Ann Intern Med. 1989;111:336-7.
18. Salmon D, Deloron P, Gaudin C, Malhotra K, Lebras J, Pocidalo JJ. Activities of pefloxacin and ciprofloxacin against experimental malaria in mice. Agents Chemother. 1990;34:2327-30.

19. Watt G, Shanks GD, Edstein MD, Pavanand K, Webster HK. Ciprofloxacin treatment of drug-resistant falciparum malaria. J Infect Dis. 1991;164:602-4.

20. Dixit SK, Mishra N, Sharma M, Singh S, Agarwal A, Awasthi SK, et al. Synthesis and in vitro antiplasmodial activities of fluoroquinolone analogs. Eur J Med Chem. 2012;51:52-9.

21. Fivelman QL, Adagu IS, Warhurst DC. Modified fixed-ratio isobologram method for studying in vitro interactions between atovaquone and proguanil or dihydroartemisinin against drug-resistant strains of Plasmodium falciparum. Antimicrob Agents Chemother. 2004;48:4097-102.

22. Berenbaum MC. A method for testing for synergy with any number of agents. J Infect Dis. 1978;137:122-30.

23. Odds FC. Synergy, antagonism, and what the chequerboard puts between them. J Antimicrob Chemother. 2003;52:1.

24. Mishra LC, Bhattacharya A, Bhasin VK. Antiplasmodial interactions between artemisinin and triclosan or ketoconazole combinations against blood stages of Plasmodium falciparum in vitro. Am J Trop Med Hyg. 2007;76:497-501.

25. Bhattacharya A, Mishra LC, Bhasin VK. In vitro activity of artemisinin in combination with clotrimazole or heat-treated amphotericin B against Plasmodium falciparum. Am J Trop Med Hyg. 2008;78:721-8.

26. Ryley JF, Peters W. The antimalarial activity of some quinolone esters. Ann Trop Med Parasitol. 1970;84:209-22.

27. Peters W. Drug resistance in Plasmodium berghei Vincke and Lips, 1948. I Chloroquine resistance Exp Parasitol. 1965;17:80-9.

28. Yadav N, Dixit SK, Bhattacharya A, Mishra LC, Sharma M, Awasthi SK, et al. Antimalarial activity of newly synthesized chalcone derivatives in vitro. Chem Biol Drug Des. 2012;80:340-7.

29. Cazelles J, Robert A, Meunier B. Alkylation of heme by artemisinin, an antimalarial drug. Comptes Rendus de l'Académie des Sciences Série IIC-Chimie. 2001;4:85-9.

30. Eckstein-Ludwig U, Webb RJ, van Goethem IDA, East JM, Lee AG, Kimura M, et al. Artemisinins target the SERCA of plasmodium falciparum. Nature. 2003;424:957-61.

31. Hartwig CL, Rosenthal AS, Dangelo J, Griffin CE, Posner GH, Cooper RA. Accumulation of artemisinin trioxane derivatives within neutral lipids of Plasmodium falciparum malaria parasites is endoperoxide-dependent. Biochem Pharmacol. 2009;77:322-36.

32. Biagini GA, Fisher N, Shone AE, Mubaraki MA, Srivastava A, Hill A, et al. Generation of quinolone antimalarials targeting the Plasmodium falciparum mitochondrial respiratory chain for the treatment and prophylaxis of malaria. Proc Nat Acad Sci USA. 2012;109:8298-303.

33. Francia MC, Striepen B. Cell division in apicomplexan parasites. Nat Rev Microbiol. 2014;12:125-36.

34. García-Estrada C, Prada CF, Fernández-Rubio C, Rojo-Vázquez F, BalañaFouce R. DNA topoisomerases in apicomplexan parasites: promising targets for drug discovery. Proc Biol Sci. 2010;277:1777-87.

35. Wells TN, Alonso PL, Gutteridge WE. New medicines to improve control and contribute to the eradication of malaria. Nat Rev Drug Discov. 2009;8:879-91.

\section{Submit your next manuscript to BioMed Central and take full advantage of:}

- Convenient online submission

- Thorough peer review

- No space constraints or color figure charges

- Immediate publication on acceptance

- Inclusion in PubMed, CAS, Scopus and Google Scholar

- Research which is freely available for redistribution 